\title{
BRST symmetry vs. Horizon condition in Yang-Mills theories
}

\author{
G. Burgio, M. Quandt, and H. Reinhardt \\ Institut für Theoretische Physik, Auf der Morgenstelle 14, 72076 Tübingen, Germany
}

\begin{abstract}
We show that in $\mathrm{SU}(2)$ Yang-Mills theories a simple relation exists between lattice gluon propagators in Coulomb and Landau gauge and discuss the physical implications of such result. In particular, the realization of the Gribov-Zwanziger confinement mechanism in Coulomb gauge, linked to dual-superconductivity, would imply that the standard BRST charge must be ill defined non perturbatively. As a consequence, the Kugo-Ojima confinement criterion, which relies on BRST charge conservation beyond perturbation theory, would not be fulfilled.
\end{abstract}

PACS numbers: 11.15.Ha, 12.38.Gc, 12.38.Aw

Keywords: Coulomb gauge, Landau gauge, Kugo-Ojima, BRST, gluon propagator 


\section{INTRODUCTION}

\section{A. Overview}

Understanding confinement in non-Abelian gauge theories has proved a major challenge for theoretical physics. How to enforce Gauß's law, which ensures gauge invariance and vanishing color charge on physical states, $Q_{c}|\Psi\rangle_{\mathrm{ph}}=0$; how to explain the discrepancy between color neutral S-matrix hadronic states and color charged fields appearing in the action; and how the IR properties of gauge fixed Green's functions should reflect confinement of gluons and quarks and non-physicality of ghost fields have been, from the very beginning, closely intertwined questions propaedeutic to the appearance of a linearly rising potential and which have spawned extensive research dating back to the '70s.

In a seminal paper, Kugo and Ojima [1] gave a criterion on the IR behavior of the ghost Green's function that ensures S-matrix states being color neutral in covariant gauges with the Faddeev-Popov (FP) action and the corresponding Becchi-Rouet-Stora-Tyutin (BRST) symmetry. According to their result, the ghost dressing function should diverge as the 4momentum goes to zero. The key assumption underlying their derivation is that a global BRST charge can be defined beyond perturbation theory, such that $Q_{\mathrm{BRST}}|\Psi\rangle_{\mathrm{ph}}=0$. Color neutrality of all S-matrix states and the disappearance of ghosts from the spectrum is then ensured by the so-called quartet mechanism [1]. Equivalently, if $Q_{c}|\Psi\rangle_{\mathrm{ph}}=0$ and the Kugo-Ojima criterion is not fulfilled, BRST symmetry must be non-perturbatively either spontaneously broken or an ill-defined concept.

A complementary approach was pioneered by Gribov [2] and later expanded by Zwanziger [3]. The basic idea is that the FP mechanism is not sufficient to define the non-Abelian partition function beyond perturbation theory. To extract a unique field along the gauge orbit the functional integral needs to be restricted, via terms which are in general non-local and are often referred to as the "Horizon condition", to the $1^{\text {st }}$ Gribov Region $\Omega$, where the FP operator is positive definite, or even further to the Fundamental Modular Region $\Lambda$, where the gauge fixing functional has only absolute minima. Obviously, any restriction on the integration domain implemented other than through the standard FP mechanism will in general break BRST symmetry. Although it can be applied also to covariant gauges, the physical implications of the Gribov-Zwanziger approach are transparent in Coulomb 
gauge. The latter has the advantage of projecting, in the Hamiltonian formulation, onto the subspace of states satisfying Gauß's law [4, 5], so that no additional assumptions to ensure $Q_{c}|\Psi\rangle_{\mathrm{ph}}=0$, as in the Kugo-Ojima approach, are needed and an explicit construction of the gauge invariant Hilbert space as in Ref. [6] can be circumvented. The Horizon condition implies in Coulomb gauge an IR diverging ghost dressing function and a vanishing static transverse gluon propagator [2]. Moreover since the inverse of the ghost dressing function can be identified with the dielectric function of the Yang-Mills vacuum [7], the latter behaves like a perfect color dia-electric medium, i.e. a dual-superconductor; a confining potential can arise via dual-Meissner effect.

\section{B. Motivations}

Approximation schemes for the set of Dyson-Schwinger equations (DSE) in QCD had been proposed already by Mandelstam in the late '70s [8], leading to the now abandoned hypothesis of infrared slavery. A first self consistent solution for the DSE was obtained by Cornwall [9] in the pinch technique framework [10], leading to a massive IR behavior of the gluon propagator. In such solution the Kugo-Ojima criterion is not fulfilled and BRST symmetry is lost non perturbatively. Only from the late '90s on alternative approaches have been developed to find solutions to the tower of DSE satisfying BRST invariance [11]. Since then, advances in functional methods for the non perturbative analysis of Yang-Mills Green's functions in Landau [12, 13] and Coulomb [14, 15] gauge on one side and the availability of large lattice simulations with improved gauge fixing techniques [16-23] on the other side have revived the interest in the IR analysis of gauge fixed Green's functions.

As mentioned above, in Landau gauge continuum methods allow in general for two types of solutions [13]. When coupled to the requirement that a global BRST charge be defined and conserved, as assumed by the Kugo-Ojima confinement criterion for the Landau ghost dressing function, they lead in any dimension to a conformal behavior of the gluon propagator in the IR, the so called "scaling" solution [11 13]. Imposing BRST symmetry to hold beyond the perturbative regime assumes implicitly that no extra care needs to be taken to restrict the functional integral beyond the FP operator. Relaxing this requirement, however, the gluon propagator can acquire a massive IR behavior [9, 13, 24, 25] while the ghost dressing function stays finite, also known as "decoupling" solution in the literature. In principle 
BRST symmetry gets explicitly broken when rendering local the terms in the action enforcing the Horizon condition, since this introduces additional ghost fields and induces modified residual transformations. Such terms should lead to a massive IR gluon propagator [24]. Alternatively, it has been argued in the literature that a vanishing gluon propagators can still be obtained in this framework [26-28] and that dimensional two condensates [29 31] are eventually responsible for the massive IR solutions as in Ref. [24].

As for the lattice, all results for the gluon and ghost in Landau gauge obtained in "standard" setups clearly favor a IR massive gluon in 2+1 and 3+1 dimensional Yang-Mills theory, while agreement with scaling is only found in $1+1$ dimensions [20, 32]. Some works applying "non-standard" lattice methods have however pointed to possible discrepancies in such picture $[33-37]$.

Whether one or the other scenario is realized in QCD is the subject of an ongoing controversy. It should be noticed that in both cases the gluon propagator violates reflection positivity and disappears from the physical spectrum anyhow [38]. Moreover, a linearly rising potential could arise in both frameworks [39]. Indeed, the issue boils down to whether color charge descends from BRST charge or, equivalently, whether in Landau gauge the Horizon condition can be neglected in favor of the standard FP mechanism.

In Coulomb gauge we are in a more comfortable position: in $3+1$ dimensions both lattice results [21] and functional methods [15, 40] show a IR vanishing static gluon transverse propagator well described by Gribov's formula [2], while the Coulomb ghost dressing function clearly diverges at zero momentum [41, 42]. The Gribov-Zwanziger mechanism is realized and a coherent picture emerges, naturally enforcing $Q_{c}|\Psi\rangle_{\mathrm{ph}}=0$ and with a direct physical interpretation in terms of dual Meissner effect [7]. As we will show below, the $2+1$ dimensional Yang-Mills theory behaves analogously.

As for the 1+1 dimensional case, the theory is topological [43] and no physical gluon exists. As a "back of the envelope" calculation shows, the Coulomb gauge propagator vanishes identically in momentum space and the corresponding FP operator can be inverted exactly, while the Landau propagator only describes pure gauge degrees of freedom [44]. An IR conformal behavior with conserved BRST charge is thus natural [43, 44]. Indeed, topological theories are the starting points for the construction of non-perturbative BRST invariance in any dimensions. Interestingly enough, lattice results, which implement some sort of Horizon condition restricting by construction to $\Omega$, and continuum calculations using 
only the FP mechanism seem to coincide in this case.

In higher dimensions however it is not clear whether the Horizon condition in Landau gauge can be reconciled with a vanishing gluon propagator. As mentioned above, different arguments have been brought up in favor [26 28] and against [9, 24, 25] it. Conventional lattice results, including direct simulations of the Kugo-Ojima function [45], are quite unambiguous in favoring the latter approach, although gauge fixing ambiguities and/or problems in the definition of the lattice gauge fields have been invoked to claim possible agreement with the former scenario [33, 34, 46], though the massive gluon IR behavior turns out to be very robust even when choosing ad-hoc prescriptions for the gauge fixing procedure to enhance the ghost propagator [36]. It is also not clear at the moment how a redefinition of the gauge fields [46] would circumvent the well known no-go theorem for a lattice BRST symmetry [47, 48]. Even if such approach would work, it would anyhow imply a deep change of paradigm for lattice gauge fixing, since on one hand a BRST invariant construction would sum over all copies in the different Gribov regions while on the other hand the standard gauge fixing procedure on the lattice follows Gribov's spirit, effectively restricting the configurations to lie within $\Omega$. There is of course a residual ambiguity in the choice of the best among all local minima found, since the explicit restriction to $\Lambda$ would require an infinite numerical precision, but on one side going to the continuum limit makes the gauge ambiguity much milder [22], while on the other side thermodynamic arguments suggest that the relevant contribution to the functional integral will anyway lie on $\partial \Lambda \cap \partial \Omega$, so that gauge ambiguities, apart from topological ones, should not play any rôle.

Since i) reliable physical input through phenomenological data is still lacking; ii) the matter turns out difficult to solve by brute force numerics, i.e. by explicitly going to the thermodynamic and/or continuum limit and iii) still waiting for a valid and viable BRST construction on the lattice, we follow here an independent approach. In dimensions higher than 1+1 a physical, although confined, gluon obviously exists and one should be able to read its degrees of freedom out of both the transverse Landau and Coulomb propagators: a relation between the two and eventually to the physical gluon degrees of freedom should exist. Moreover if Gribov's prescription to restrict the functional integral is the physically sound one in Coulomb gauge, since it seems to agree with dual superconductivity, one would expect the Gribov mass appearing there [2, 21] to be linked to some physical quantity, i.e it should effectively be gauge invariant. This mass should show up in Landau, interpolating 
[49] and covariant gauges [50] and be related to the effective IR gluon mass [9].

In $d+1$ dimensions the most natural Ansatz to relate the static transverse gluon propagator in Coulomb gauge and the transverse gluon propagator in Landau gauge is via a one to one correspondence between the variables they must be function of, namely $\vec{p}^{2}$, the natural $\mathrm{O}(d)$ invariant in the static Hamiltonian Coulomb gauge picture, and $p^{2}$, its $\mathrm{O}(d+1)$ covariant counterpart in euclidian lattice Landau gauge. We therefore assume that in $2+1$ and $3+1$ dimensions by just relating monotonically the $d$-and $d+1$-momentum scales the two propagators will coincide. Based on this simple Ansatz we will show that i) there is a one to one correspondence between the lattice results for the IR vanishing static Coulomb gluon propagator, realizing Gribov's original Horizon condition [2] and the IR massive Landau propagator and ii) that within this correspondence the Coulomb gauge Gribov mass exactly coincides with the Landau gauge effective IR gluon mass. Should the uniqueness of the massive behavior for lattice gluon propagator in Landau gauge be confirmed, one could interprete the loss of BRST symmetry beyond perturbation theory that would descend from it as a consequence of the dual-superconductor mechanism in QCD.

\section{RESULTS}

We calculate the transverse gluon propagator in Coulomb and Landau gauge in $2+1$ and 3+1 dimensional SU(2) Yang-Mills theory on configurations generated via MC simulations on lattices up to $64^{3}$ and $32^{4}$ and $\beta$ between 3-12 and 2.15-2.6 respectively, using a heath bath plus over-relaxation algorithm. The scale is set re-expressing the lattice spacing in units of the string tension [17, 51], which we fix to $\sigma=(440 \mathrm{MeV})^{2}$ also in $2+1$ dimensions.

\section{A. Coulomb propagator}

In Coulomb gauge renormalization issues on the lattice have only been recently clarified in Ref. [21, 52]. We extend here our procedure to $2+1$ dimensions and improve our gauge fixing algorithm.

For each fixed $\beta$ we define the gluon propagator $D_{i j}^{a b}(p)$ as the Fourier transform of the gluon two-point function:

$$
D_{i j}^{a b}(p)=\left\langle\widetilde{A}_{i}^{a}(k) \widetilde{A}_{j}^{b}(-k)\right\rangle_{U}=\delta^{a b} \delta_{i j} D_{\beta}(p), \quad p_{\mu}=p\left(k_{\mu}\right)=\frac{2}{a} \sin \left(\frac{\pi k_{\mu}}{L}\right) .
$$


Here $\widetilde{A}_{\mu}^{a}(k)$ is the Fourier transform of the lattice gauge potential $A_{\mu}^{a}(x+\hat{\mu} / 2)$, defined as:

$$
A_{\mu}(x+\hat{\mu} / 2)=\frac{1}{2 i}\left(U_{\mu}(x)-U_{\mu}(x)^{\dagger}\right)
$$

$a$ is the lattice spacing, $p=\left(\vec{p}, p_{0}\right)$ denotes the four-momentum and $k_{\mu} \in(-L / 2,+L / 2]$ are the integer-valued lattice momenta. We always select spatial indices $\vec{k}$ satisfying a cylinder cut [16, 41] to minimize violations of rotational invariance. Time-like momenta $p_{0}$ are unconstrained.

For each MC generated configuration we first fix one of the $2^{d}$ flip sectors [19], where $d$ are the spatial dimensions, and then fix Coulomb gauge maximizing separately for every time slice $t$ the gauge functional:

$$
F_{g}(t)=\frac{1}{6 L^{3}} \sum_{\vec{x}, i} \operatorname{Tr} U_{i}^{g}(\vec{x}, t), \quad U_{i}^{g}(\vec{x}, t)=g(\vec{x}, t) U_{i}(\vec{x}, t) g^{\dagger}(\vec{x}+\hat{i}, t)
$$

with respect to local gauge transformations $g(\vec{x}, t) \in \mathrm{SU}(2)$, employing a simulated annealing plus over-relaxation algorithm. The local maxima of $F_{g}(t)$ satisfy for each fixed $t$ the differential lattice Coulomb gauge transversality condition for the gauge potentials:

$$
\partial_{i} A_{i}^{g}(\vec{x}, t)=A_{i}^{g}(\vec{x}+\hat{i} / 2, t)-A_{i}^{g}(\vec{x}-\hat{i} / 2, t)=0
$$

We choose the best $F_{g}(t)$ out of $n_{c}$ random gauge copy for each time slice $t$ separately, where usually $n_{c}=5$, and combine them into the best copy of the whole configuration for the flip sector chosen. We now go to the next sector and repeat the procedure. At the end we chose the sector with the highest global functional $F_{g}=\sum_{t} F_{g}(t)$ as the best copy of the configuration. The number of total recursions is $2^{d} L n_{c}$. Increasing $n_{c}$ leads of course to a better gauge fixing. However, going to higher volumes, one might consider to increase $n_{c}$ only for the sectors with the highest $F_{g}[19]$.

To extract the $p_{0}$ dependence we need to fix the time gauge $g(t)$. We choose here the integrated Polyakov gauge prescription proposed in Ref. [21, 52]:

$$
u(t)=\frac{1}{L^{3}} \sum_{\vec{x}} U_{0}(\vec{x}, t) \rightarrow \text { const. }
$$

corresponding in the continuum to

$$
0=\int d^{3} x \partial_{\mu} A_{\mu}(\vec{x}, t) \Rightarrow \partial_{0} \int d^{3} x A_{0}(\vec{x}, t)=0
$$


To achieve this, we define

$$
\hat{u}(t)=\frac{u(t)}{\sqrt{\operatorname{Det}[u(t)]}} \in S U(2) .
$$

Periodic boundary conditions in $t$ make $\operatorname{Tr} \prod_{t} \hat{u}(t)=\operatorname{Tr} P$ invariant under $g(t)$. Eq. (5) can thus be fixed recursively through

$$
g(t) \hat{u}(t) g^{\dagger}(t+1)=\tilde{u} \equiv P^{1 / L}
$$

up to a global gauge $g(0)$ satisfying $[g(0), P]=0$. We can choose $g(0)=\mathbb{1}$.

The main observation, made in Ref. [21, 52] for the $3+1$ dimensional case and which we find here to hold in $2+1$ dimensions as well, is that the bare gluon propagator factorizes as:

$$
D_{\beta}\left(|\vec{p}|, p_{0}\right)=\frac{f_{\beta}(|\vec{p}|)}{|\vec{p}|^{2}} \frac{g_{\beta}(z)}{1+z^{2}}, \quad z=\frac{p_{0}}{|\vec{p}|} .
$$

The function $g_{\beta}(z)$ will in general depend on the temporal gauge $g(t)$. However the static propagator, defined as

$$
D_{\beta}(|\vec{p}|)=\int_{-\infty}^{\infty} \frac{d p_{0}}{2 \pi} D_{\beta}\left(|\vec{p}|, p_{0}\right)=\frac{f_{\beta}(|\vec{p}|)}{\pi|\vec{p}|} \int_{0}^{\infty} d z \frac{g_{\beta}(z)}{1+z^{2}},
$$

will be independent of such choice. As shown in Ref. [21, 52] lattice cutoffs to the integral in Eq. (10) cause scaling violations; as a viable [53] alternative to the lattice Hamiltonian limit [54] we define $D_{\beta}(|\vec{p}|)$ directly from $f_{\beta}(|\vec{p}|)$, extracting it from the data after dividing out $g_{\beta}(z)$ and averaging over $p_{0}$ :

$$
D_{\beta}(|\vec{p}|):=\frac{f_{\beta}(|\vec{p}|)}{|\vec{p}|} \propto|\vec{p}| \sum_{p_{0}} D_{\beta}\left(|\vec{p}|, p_{0}\right) \frac{1+z^{2}}{g_{\beta}(z)} .
$$

We obtain in this way a multiplicative renormalizable $D_{\beta}(|\vec{p}|), D_{\mu}(|\vec{p}|)=Z(\beta, \mu) D_{\beta}(|\vec{p}|)$. We choose $\mu=\infty$, i.e. we fix the overall normalization such that $\lim _{|\vec{p}| \rightarrow \infty}|\vec{p}| D_{\mu}(|\vec{p}|)=1$.

In $2+1$ dimensions a Gribov like IR leading behavior:

$$
G(|\vec{p}|)=\frac{|\vec{p}|}{\sqrt{|\vec{p}|^{4}+M^{4}}}
$$

plus corrections of the type:

$$
\tilde{G}(|\vec{p}|)=\frac{|\vec{p}|^{1+\alpha}}{\sqrt[\beta]{|\vec{p}|^{(2+\alpha) \beta}+\mu^{(2+\alpha) \beta}}}
$$

describes the data extremely well. To account for the intermediate momentum region different choices for the sub-leading terms other than Eq. (13) can be made, the only constraints 
being asymptotic behaviors $|\vec{p}|^{-1}$ in the UV and $o(|\vec{p}|)$ in the IR. Lacking any theoretical input further restricting the functional form is beyond our scope. In Fig. 1 we show $D_{C}(|\vec{p}|)=|\vec{p}|^{-1} D_{\mu}(|\vec{p}|)$, which nicely extrapolates to a constant in the IR, together with the fit to $|\vec{p}|^{-1}(c G(|\vec{p}|)+\tilde{c} \tilde{G}(|\vec{p}|))$. To improve readability versus the $3+1$ dimensional propagator we have normalized the data to $3.5|\vec{p}|^{-2}$ in the UV. We find $M=4.6(4) \mathrm{GeV}$ and $0.2 \leq \chi^{2} /$ d.o.f. $\leq 0.6$, depending on the choice of the sub-leading terms, our main source of error.

In $3+1$ dimensions $D_{\mu}(|\vec{p}|)$ is well described by Gribov's formula alone [21]. In Fig. 11 we show $D_{C}(|\vec{p}|)=|\vec{p}|^{-1} D_{\mu}(|\vec{p}|)$ and its fit to $|\vec{p}|^{-1} G(|\vec{p}|)$ as in Eq. (12); we obtain $M=0.856(8)$ $\mathrm{GeV}$ with $\chi^{2} /$ d.o.f. $=1.6$. In Ref. [21] the deep IR data slightly deviated from a constant behavior, resulting in a $\chi^{2} /$ d.o.f. $=3.3$ for the same fit. These deviations are here, with increased volume and improved gauge fixing, considerably smaller, halving the $\chi^{2} /$ d.o.f. value. We therefore decided to ignore possible sub-leading corrections as in Eq. (13) for the time being. Only a study on larger volumes and closer to the continuum limit, in the spirit of the Landau gauge analysis of Ref. [22, 23, 55], could clarify the situation. We also stress that no logarithmic corrections are necessary to describe the data in the UV. Although this is expected in $2+1$ dimensions, it is a highly non trivial result in the $3+1$ dimensional case, since one loop calculations for $D_{\beta}\left(|\vec{p}|, p_{0}\right)$ give an explicit anomalous dimension [56]. However, contrary to covariant gauges, neither higher orders are under control nor a leadinglog re-summation is viable. Cancellations when going from the one loop full propagator to the full static propagator are therefore in principle possible.

As a last remark, both in $2+1$ and $3+1$ dimensions the asymptotic behaviors of $D_{\mu}(|\vec{p}|)$ are in agreement with the results of the IR and UV analysis of the DSE in the Hamiltonian approach of Ref. [15, 57].

\section{B. Landau propagator}

The lattice gluon propagator in Landau gauge $D_{L}(p)$ has been the subject of extensive research; we refer the interested reader to the relevant literature regarding restoration of rotational invariance, gauge copy effects, continuum and thermodynamic limits etc. [16-20]. Here we simply apply the by now well established methods developed in the above references. In particular, we basically follow Ref. [18, 19]. 


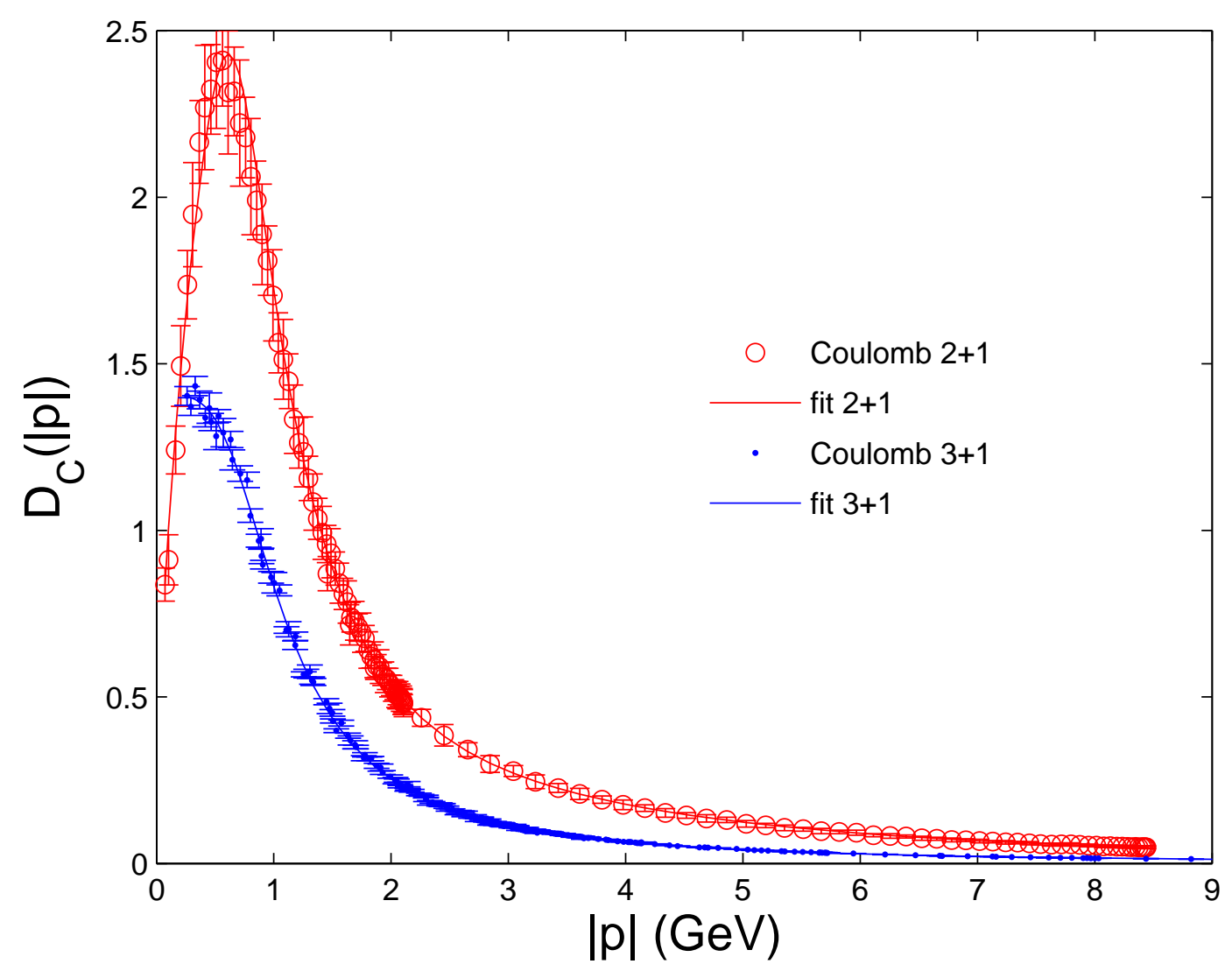

FIG. 1. $D_{C}(|\vec{p}|)$ and fits in $2+1$ and $3+1$ dimensions.

The results for $2+1$ and $3+1$ dimensions, normalized such that the peaks coincide with those of Fig. 1, are shown in Fig. 2 and agree with all known previously published results.

\section{Landau vs. Coulomb}

On trivial dimensional grounds, based on their tree level UV behavior, it is obvious that $D_{L}(p)$ should be compared to $D_{C}(|\vec{p}|)=|\vec{p}|^{-1} D_{\mu}(|\vec{p}|)$. For both $2+1$ and $3+1$ dimensions a simple inspection of the Landau gauge transverse propagator plot against $p=\sqrt{|\vec{p}|^{2}+p_{0}^{2}}$ given in Fig. 2 shows striking similarities with $D_{C}(|\vec{p}|)$ plotted against $|\vec{p}|$, as in Fig. 1) The two curves almost coincide, up to a faster fall off of $D_{L}(p)$ in the UV. In fact, it simply looks as if the Landau 4-momentum scale $p$ is stretched by some factor when compared to the Coulomb momentum scale $|\vec{p}|$. For a quantitative comparison, motivated by this observation, we make the simple Ansatz $D_{L}(p) \equiv D_{C}\left(p_{L}(p)\right)$, where $p_{L}(p)=p \rho(p / \Lambda)$ 


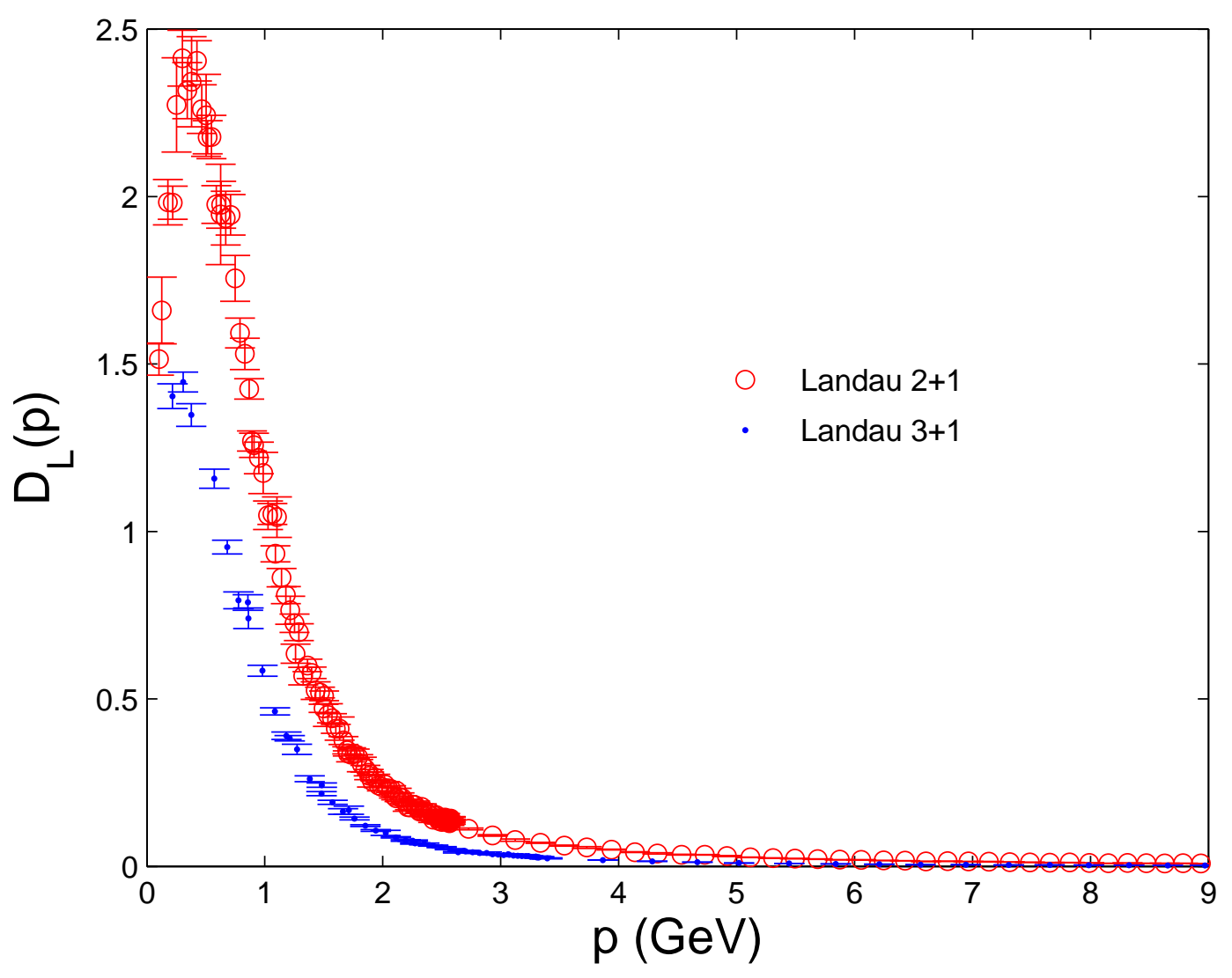

FIG. 2. $D_{L}(p)$ in $2+1$ and $3+1$ dimensions.

is strictly monotonous and $\rho(p / \Lambda)$ is a dimensionless function interpolating between two constants $\rho_{\mathrm{IR}}$ and $\rho_{\mathrm{UV}}$ in $2+1$ dimensions, where the anomalous dimension vanishes, and between a constant $\rho_{\mathrm{IR}}$ and $\rho_{\mathrm{UV}} \log ^{\gamma / 2} p^{2} / \Lambda_{\mathrm{QCD}}^{2}$ in $3+1$ dimensions. We use for $\rho(p / \Lambda)$ a ratio of rational functions of the same order, $\rho(p / \Lambda)=R_{n}\left(p^{2} / \Lambda^{2}\right) / R_{d}\left(p^{2} / \Lambda^{2}\right)$, corrected in $3+1$ dimensions by $\log ^{\gamma / 2} R_{l}\left(p^{2} / \Lambda_{\mathrm{QCD}}^{2}\right), R_{l}$ being also a rational function. Polynomials of degree $\leq 3$ for $R_{n, d, l}(x)$ are sufficient for our scope. In $3+1$ dimensions shuffling the $p$ dependence inside or outside the log is somewhat arbitrary. Indeed, a form of the type $\rho\left(p / \Lambda_{\mathrm{QCD}}\right)=\rho_{\mathrm{IR}} \log ^{\gamma / 2} R_{l}\left(p^{2} / \Lambda_{\mathrm{QCD}}^{2}\right)$ is already sufficient, so that in both $2+1$ and $3+1$ dimensions one single mass parameter, $\Lambda$ or $\Lambda_{\mathrm{QCD}}$ is needed to describe $\rho$. A certain level of arbitrariness is also present in the normalization of the highest and lowest coefficient of $R_{n, d, l}(x)$ as well as in their degree. The errors quoted below reflect all such, partly correlated, uncertainties, which will in particular show up in the value of $\Lambda$ and $\Lambda_{\mathrm{QCD}}$. However, lacking further theoretical input, pinning down $\rho(p)$ to a precise functional form goes be- 
yond the scope of this work. We have fitted both $|\vec{p}| D_{C}(|\vec{p}|)$ and $p_{L}(p) D_{L}\left(p_{L}(p)\right)$ at the same time to Eq. (12) (plus Eq. (13) in $2+1$ dimensions). $\rho(p)$ as obtained from the fits is shown in Fig. 3, while the resulting comparison between $D_{C}(|\vec{p}|)$ vs. $D_{L}(p)$ plotted against $p_{L}(p)$ in $2+1$ and $3+1$ dimensions is given in Fig. 4 and Fig. 5. Numerically, we obtain $\rho_{\mathrm{IR}}=1.44(14), \rho_{\mathrm{UV}}=2.610(15), \Lambda=3.0(3) \mathrm{GeV}$ and $\chi^{2} /$ d.o.f. $=1.2$ in $2+1$ dimensions, $\rho_{\mathrm{IR}}=1.255(15), \rho_{\mathrm{UV}}=1.49(1), \gamma=0.52(3)$ and $\Lambda_{\mathrm{QCD}}=1.05(15) \mathrm{GeV}$ with $\chi^{2} /$ d.o.f. $=1.6$ in 3+1 dimensions; the fitted values for all parameters in Eq. (12) and Eq. (13), particularly for $M$, exactly coincide with those of Section IA Moreover our $3+1$ dimensional value for $M=0.856(8) \mathrm{GeV}$ is in excellent agreement with recent $\mathrm{SU}(3)$ estimates of the IR mass in Landau gauge which explicitely include a dimensional two condensate in the analysis [58].

It is obvious that there is no way to relate $D_{C}(|\vec{p}|)$ to a scaling, IR vanishing $D_{L}(p)$ while also accounting for the UV region, since a monotonous mapping between the momenta could never do the job. One could of course object that the choice, in our opinion natural, to compare the Landau propagator with $D_{C}(|\vec{p}|)$ already shuts the doors to any scaling behavior. One could therefore attempt to compare $D_{L}(p)$ directly to $D_{\mu}(|\vec{p}|)$ again via a monotonous mapping of the momenta, e.g. $D_{L}(p) \equiv D_{\mu}\left(\bar{p}_{L}(p)\right)$, to check if an agreement to the same level of accuracy as in our analysis could be reached. In this case the function $\bar{p}_{L}(p)$ should behave up to dimensionful constants like $\propto p^{\kappa}$ in the IR and $\propto p^{2}$ (plus logarithms in $3+1$ dimensions) in the UV, i.e. $\bar{p}_{L}(p)$ would in principle need two new mass scales, besides the one for the intermediate momentum region, to account for the IR and UV behavior. As discussed above and as can be inferred from Fig. 3, this should be compared to the present analysis, where the only mass scale needed in $\rho(p)$ basically coincides with the Gribov mass both in $2+1$ and $3+1$ dimensions, even though its exact value will depend on the, still arbitrary, parameterization of $\rho(p)$. Moreover, that the Gribov parameter $M$ extracted both from the lattice Coulomb and Landau gauge gluon transverse degrees of freedom coincide to such degree of precision constitutes in our opinion a highly non trivial result supporting a physical interpretation for the dynamically generated gluon mass. Of course the points raised above do not forbid in principle the existence of a mapping between the Gribov-like Coulomb propagator and a scaling like solution in Landau gauge, but lacking any theoretical arguments and as long as one can neither obtain a scaling solution on the lattice nor a solution obtained with continuum methods is available to sufficient degree of precision, any analogous quantitative check as the one performed in this paper to prove or 
disprove such correspondence will remain impossible.

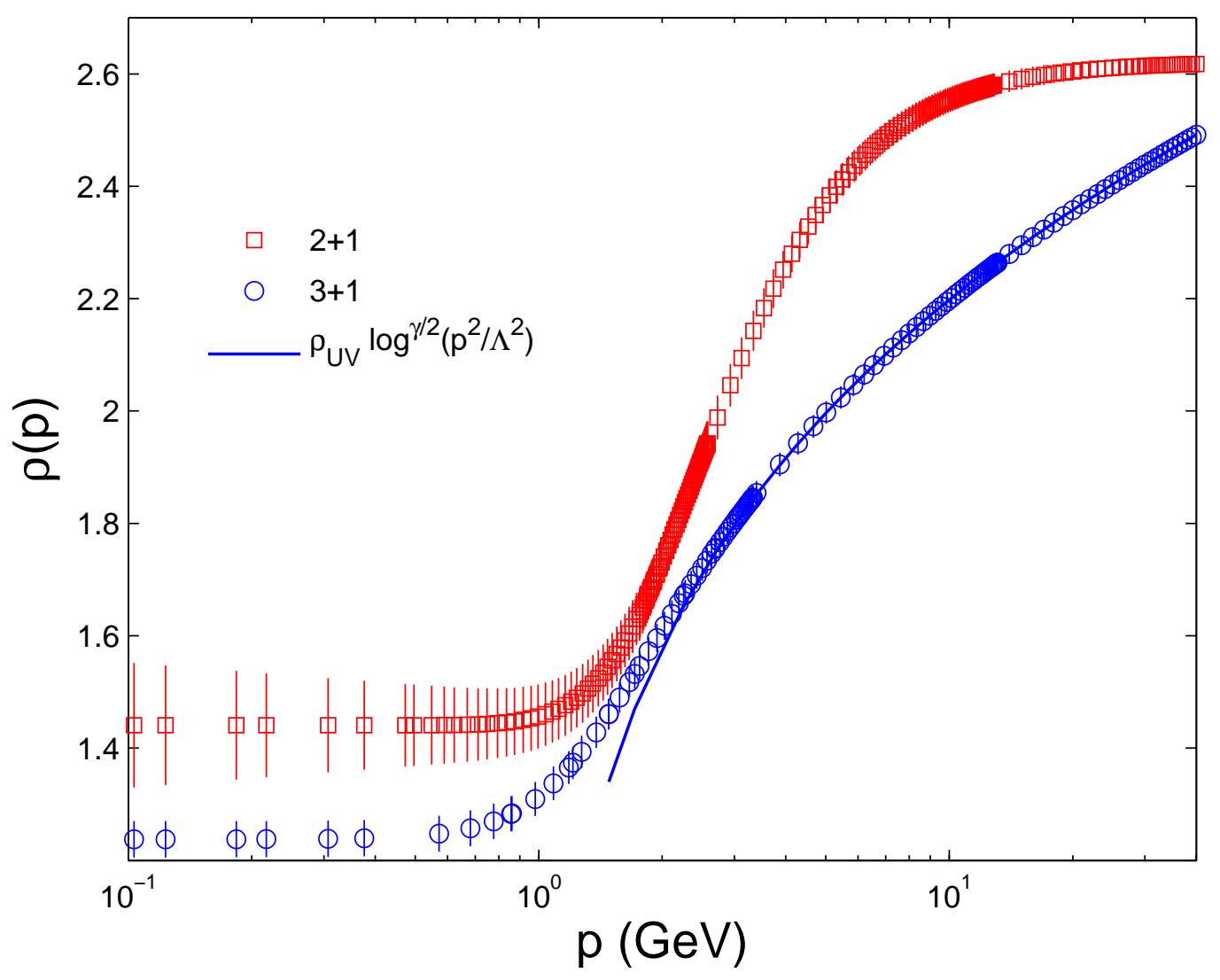

FIG. 3. $\rho(p)$ in $2+1$ and $3+1$ dimensions, the latter compared to the perturbative asymptotic behavior.

\section{CONCLUSIONS}

We have shown that in $2+1$ and $3+1$ dimensional Yang-Mills theory, where physical gluons propagate, a natural and simple relation exists between the lattice results for the gluon-gluon Green's functions in two different gauges, i.e. the IR finite transverse Landau propagator $D_{L}(p)$ and the IR vanishing static transverse Coulomb propagator $D_{\mu}(|\vec{p}|)$. Indeed, the two are equivalent if one simply rescales the momenta. i.e. $D_{L}(p)=p_{L}(p)^{-1} D_{\mu}\left(p_{L}(p)\right)$ where $p_{L}(p)=p \rho(p)$ and $\rho(p)$ is given in Fig. 3. $D_{\mu}(|\vec{p}|)$ agrees with the Gribov-Zwanziger scenario and has a natural interpretation within the dual-superconductor picture of confinement [7]. This corroborates a monopole condensation interpretation of the gluon massive solution 


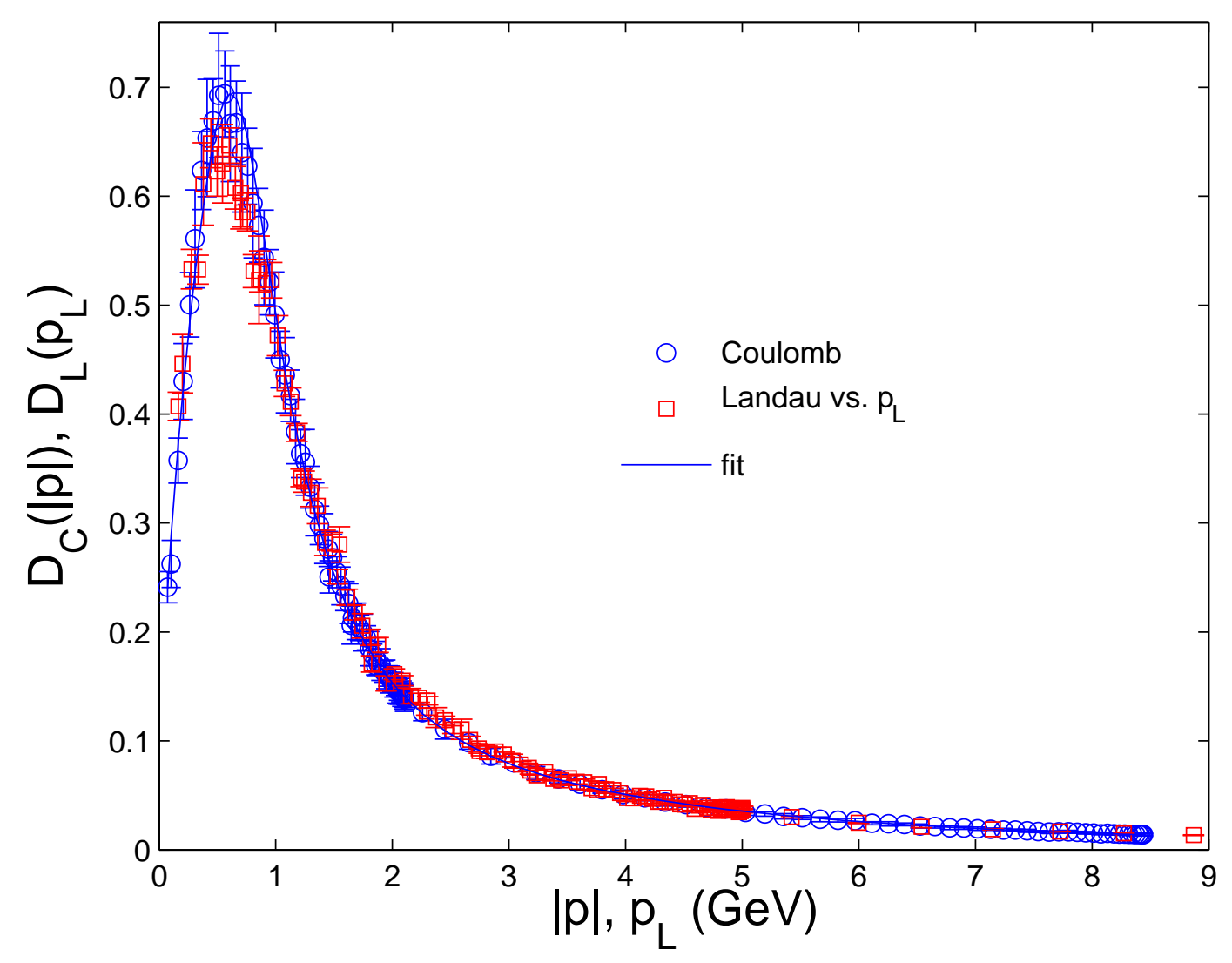

FIG. 4. $D_{C}(|\vec{p}|), D_{L}\left(p_{L}(p)\right)$ and fit in $2+1$ dimensions.

in Landau gauge as well, making, as long as no scaling like solution can be found on the lattice, the non perturbative loss of BRST symmetry more bearable. Whether the static Coulomb gluon itself coincides with the Yang-Mills field's physical degrees of freedom is still an open issue [59, 60]. Should the uniqueness of the IR massive gluon behavior on the lattice be confirmed, it would suggests that in covariant gauges in more than one spatial dimension the Gribov-Zwanziger [2, 3] term should be explicitly included in the action, BRST symmetry would be non-perturbatively broken and the Kugo-Ojima criterion [1] could not be fulfilled. Indeed an effectively massive gluon propagator is natural in a theory where monopoles condense, as can be explicitly seen in the Georgi-Glashow model [61, 62]; dual-superconductivity is expected to work in a similar way to confine gluons, albeit with a dynamically generated scalar boson [63]. Indeed, a topological origin for dynamic mass generation in QCD has been argued long time ago in Ref. [64] and offers a natural interpretation of the pinch technique result [9, 10]. In topological theories on the other 


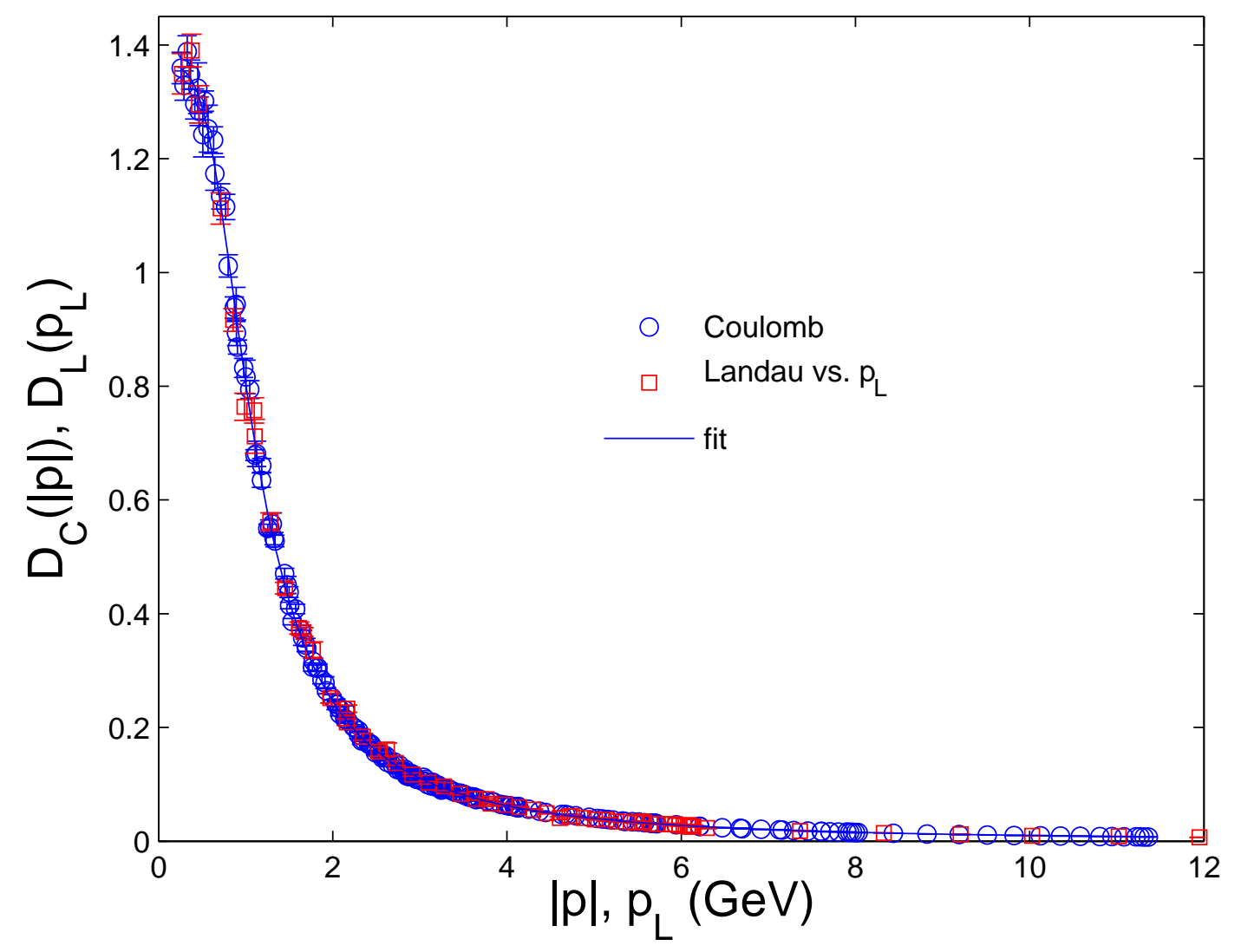

FIG. 5. $D_{C}(|\vec{p}|), D_{L}\left(p_{L}(p)\right)$ and fit in $3+1$ dimensions.

hand, where the physical Coulomb propagator should identically vanishes, a IR conformal confinement with a non perturbatively conserved BRST charge can be realized. The ChernSimons and BF theories will be interesting cross-checks to confirm the different mechanism between topological and QCD like theories.

\section{ACKNOWLEDGMENTS}

We would like to thank Peter Watson and Jan M. Pawlowski for stimulating discussions. This work was partly supported by DFG under the contract DFG-Re856/6-3.

[1] T. Kugo and I. Ojima, Prog. Theor. Phys. Suppl. 66, 1 (1979).

[2] V. N. Gribov, Nucl. Phys. B139, 1 (1978). 
[3] D. Zwanziger, Nucl. Phys. B485, 185 (1997), hep-th/9603203.

[4] H. Reinhardt and P. Watson, Phys. Rev. D79, 045013 (2009), 0808.2436.

[5] H. Reinhardt and W. Schleifenbaum, Annals Phys. 324, 735 (2009), 0809.1764.

[6] G. Burgio, R. De Pietri, H. A. Morales-Tecotl, L. F. Urrutia, and J. D. Vergara, Nucl. Phys. B566, 547 (2000), hep-lat/9906036.

[7] H. Reinhardt, Phys. Rev. Lett. 101, 061602 (2008), 0803.0504.

[8] S. Mandelstam, Phys. Rev. D20, 3223 (1979).

[9] J. M. Cornwall, Phys. Rev. D26, 1453 (1982).

[10] D. Binosi and J. Papavassiliou, Phys. Rept. 479, 1 (2009), 0909.2536.

[11] L. von Smekal, R. Alkofer, and A. Hauck, Phys. Rev. Lett. 79, 3591 (1997), hep-ph/9705242.

[12] C. S. Fischer and J. M. Pawlowski, Phys. Rev. D75, 025012 (2007), hep-th/0609009.

[13] C. S. Fischer, A. Maas, and J. M. Pawlowski, Annals Phys. 324, 2408 (2009), 0810.1987.

[14] A. P. Szczepaniak and E. S. Swanson, Phys. Rev. D65, 025012 (2002), hep-ph/0107078.

[15] C. Feuchter and H. Reinhardt, Phys. Rev. D70, 105021 (2004), hep-th/0408236.

[16] D. B. Leinweber et al. (UKQCD), Phys. Rev. D60, 094507 (1999), hep-lat/9811027.

[17] J. C. R. Bloch, A. Cucchieri, K. Langfeld, and T. Mendes, Nucl. Phys. B687, 76 (2004), hep-lat/0312036.

[18] I. L. Bogolubsky et al., Phys. Rev. D74, 034503 (2006), hep-lat/0511056.

[19] I. L. Bogolubsky et al., Phys. Rev. D77, 014504 (2008), 0707.3611.

[20] A. Cucchieri and T. Mendes, Phys. Rev. Lett. 100, 241601 (2008), 0712.3517.

[21] G. Burgio, M. Quandt, and H. Reinhardt, Phys. Rev. Lett. 102, 032002 (2009), 0807.3291.

[22] I. L. Bogolubsky, E. M. Ilgenfritz, M. Muller-Preussker, and A. Sternbeck (2009), 0912.2249.

[23] V. G. Bornyakov, V. K. Mitrjushkin, and M. Muller-Preussker (2009), 0912.4475.

[24] D. Dudal, J. A. Gracey, S. P. Sorella, N. Vandersickel, and H. Verschelde, Phys. Rev. D78, 065047 (2008), 0806.4348.

[25] K.-I. Kondo (2009), 0904.4897.

[26] D. Zwanziger (2009), 0904.2380.

[27] D. Zwanziger, Nucl. Phys. B399, 477 (1993).

[28] D. Zwanziger (2010), 1003.1080.

[29] R. Akhoury and V. I. Zakharov, Phys. Lett. B438, 165 (1998), hep-ph/9710487. 
[30] G. Burgio, F. Di Renzo, G. Marchesini, and E. Onofri, Phys. Lett. B422, 219 (1998), hep$\mathrm{ph} / 9706209$.

[31] P. Boucaud et al., JHEP 04, 006 (2000), hep-ph/0003020.

[32] A. Cucchieri and T. Mendes, Phys. Rev. D78, 094503 (2008), 0804.2371.

[33] A. Sternbeck and L. von Smekal (2008), 0811.4300.

[34] A. Maas, J. M. Pawlowski, D. Spielmann, A. Sternbeck, and L. von Smekal (2009), 0912.4203.

[35] P. J. Silva and O. Oliveira, Phys. Rev. D74, 034513 (2006), hep-lat/0511043.

[36] A. Maas (2009), 0907.5185.

[37] O. Oliveira and P. J. Silva (2009), 0911.1643.

[38] A. Cucchieri, T. Mendes, and A. R. Taurines, Phys. Rev. D71, 051902 (2005), heplat/0406020.

[39] J. Braun, H. Gies, and J. M. Pawlowski, Phys. Lett. B684, 262 (2010), 0708.2413.

[40] D. Epple, H. Reinhardt, and W. Schleifenbaum, Phys. Rev. D75, 045011 (2007), hepth/0612241.

[41] A. Voigt, E.-M. Ilgenfritz, M. Muller-Preussker, and A. Sternbeck, PoS LAT2007, 338 (2007), 0709.4585 .

[42] M. Quandt, G. Burgio, S. Chimchinda, and H. Reinhardt, PoS CONFINEMENT8, 066 (2008), 0812.3842.

[43] E. Witten, Commun. Math. Phys. 121, 351 (1989).

[44] D. Birmingham, M. Blau, M. Rakowski, and G. Thompson, Phys. Rept. 209, 129 (1991).

[45] S. Furui and H. Nakajima, Phys. Rev. D70, 094504 (2004).

[46] L. von Smekal, A. Jorkowski, D. Mehta, and A. Sternbeck, PoS CONFINEMENT8, 048 (2008), 0812.2992.

[47] H. Neuberger, Phys. Lett. B175, 69 (1986).

[48] H. Neuberger, Phys. Lett. B183, 337 (1987).

[49] A. Cucchieri, A. Maas, and T. Mendes, Mod. Phys. Lett. A22, 2429 (2007), hep-lat/0701011.

[50] A. Cucchieri, T. Mendes, and E. M. S. Santos, Phys. Rev. Lett. 103, 141602 (2009), 0907.4138.

[51] M. J. Teper, Phys. Rev. D59, 014512 (1999), hep-lat/9804008.

[52] G. Burgio, M. Quandt, and H. Reinhardt, PoS CONFINEMENT8, 051 (2008), 0812.3786.

[53] Y. Nakagawa, A. Nakamura, T. Saito, and H. Toki (2009), 0911.2550.

[54] G. Burgio and al. (TrinLat), Phys. Rev. D67, 114502 (2003), hep-lat/0303005. 
[55] A. Cucchieri and T. Mendes, PoS LAT2007, 297 (2007), 0710.0412.

[56] P. Watson and H. Reinhardt, Phys. Rev. D76, 125016 (2007), 0709.0140.

[57] C. Feuchter and H. Reinhardt, Phys. Rev. D77, 085023 (2008), 0711.2452.

[58] D. Dudal, O. Oliveira, and N. Vandersickel (2010), 1002.2374.

[59] X.-S. Chen, X.-F. Lu, W.-M. Sun, F. Wang, and T. Goldman, Phys. Rev. Lett. 100, 232002 (2008), 0806.3166.

[60] X.-S. Chen, W.-M. Sun, X.-F. Lu, F. Wang, and T. Goldman, Phys. Rev. Lett. 103, 062001 (2009), 0904.0321.

[61] A. M. Polyakov, Nucl. Phys. B120, 429 (1977).

[62] G. V. Dunne, I. I. Kogan, A. Kovner, and B. Tekin, JHEP 01, 032 (2001), hep-th/0010201.

[63] G. 't Hooft, Nucl. Phys. B79, 276 (1974).

[64] J. M. Cornwall, Nucl. Phys. B157, 392 (1979). 